\title{
ABVE Regimen
}

National Cancer Institute

\section{Source}

National Cancer Institute. ABVERegimen. NCI Thesaurus. Code C67162.

A regimen containing doxorubicin hydrochloride, bleomycin sulfate, vincristine sulfate and etoposide used in combination with radiation therapy for the treatment of low-risk, childhood Hodg kin lymphoma. 